\title{
LEITLINIE ZUR PRÄVENTION UND THERAPIE DER HYPOGLYKÄMIE BEI NEUGEBORENEN AB 35+0 SCHWANGERSCHAFTSWOCHEN AUF DER WOCHENBETTSTATION
}

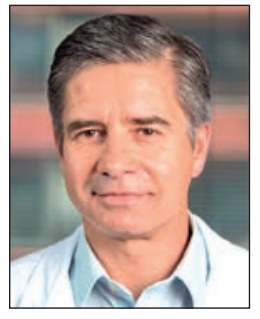

Sven Schulzke

\section{Was ist neu?}

Interventionsgrenze für neonatale Hypoglykämie neu $<2.6 \mathrm{mmol} / \mathrm{L}$ (zuvor $<2.5 \mathrm{mmol} / \mathrm{L}$ )

Dextrose-Gel $40 \%$ als ergänzende Massnahme für Prävention und Therapie

Berücksichtigung neuer Studien zur neurologischen Entwicklung nach Hypoglykämie

Neue Daten zum Spontanverlauf des Blutzuckers in den ersten Lebenstagen bei gesunden Neugeborenen

\section{Einleitung}

Ziel dieser Leitlinie ist es, Empfehlungen für die Prophylaxe und Therapie der Hypoglykämie bei Neugeborenen ab 35+0 Schwangerschaftswochen (SSW) im Gebärsaal und auf der Wochenbettstation zu geben. Dies entspricht Stationen der neonatologischen Basisversorgung Level I gemäss der Kommission zur Akkreditierung neonatologischer Stationen (CANU) der Schweizerischen Gesellschaft für Neonatologie $(\mathrm{SGN})^{1)}$. Diese Leitlinie beinhaltet nicht die Behandlung von Frühgeborenen < 35 SSW und kranken Termingeborenen, die in eine Neonatologie der Stufe Ila, Ilb oder III gemäss CANU gehören ${ }^{11}$.

\section{Hintergrund}

Bei der Geburt finden verschiedene Stoffwechselanpassungen statt, um den Übergang von einer Glukosebereitstellung über die Plazenta und fetalen Glykogensynthese hin zur unabhängigen Glukoseproduktionund regulation durch das Neugeborene sicherzustellen. Die endogenen Glukagon- und Katecholaminspiegel des Kindes steigen um das 3-5fache an, was eine Glykogenolyse bewirkt, die für die ersten Lebensstunden zu einem ausgeglichenen Blutzucker führt. Die Ausschüttung von Wachstumshormon und Cortisol begünstigt des Weiteren die Glukoneogenese, insbesondere jenseits der ersten Lebensstunden. Gleichzeitig sinken die Insulinspiegel. Damit Glykogenolyse und Glukoneogenese entsprechend anlaufen können, braucht es die zugehörigen Enzyme und Substrate wie Glykogen, Fett und Aminosäuren²-5).
Selbst bei optimaler postnataler Anpassung kann der Blutzuckerspiegel bei gesunden Neugeborenen in- nerhalb der ersten Lebensstunden niedrig sein. Er steigt danach üblicherweise langsam aber stetig an und bleibt stabil bis etwa 48 Lebensstunden (Mittelwert (Standardabweichung) $3.3(0.6) \mathrm{mmol} / \mathrm{L}$ ), bevor er weiter steigt und am vierten Lebenstag ein neues Plateau erreicht (Mittelwert (Standardabweichung) $4.6(0.7) \mathrm{mmol} / \mathrm{L})^{6}$. Allerdings kann bei bis zu einem Drittel der gesunden Termingeborenen mindestens eine Episode von Hypoglykämie unter $2.6 \mathrm{mmol} / \mathrm{L}$ innerhalb der ersten Lebenstage beobachtet werden, insbesondere in den ersten 12 Lebensstunden ${ }^{6}$.

Glukose ist das primäre Substrat des zerebralen Energiestoffwechsels. Neugeborene haben wegen des relativ hohen Gewichts ihres Gehirns im Verhältnis zum Körpergewicht einen besonders hohen, gewichtsadaptierten Glukosebedarf. Das Gehirn kann auch Laktat oder Ketone als Energiequelle nutzen, die im Falle von Lipolyse produziert werden ${ }^{7)}$; daher ist es nicht notwendig, bei gesunden Termingeborenen routinemässig serielle Blutzuckerbestimmungen durchzuführen. Im Gegensatz dazu gibt es Neugeborene, die ein erhöhtes Risiko für neonatale Hypogykämien haben, z.B. nach perinatalem Stress oder bei maternalem Diabetes. Letzteres führt überwiegend durch einen relativen Hyperinsulinismus zu einer transienten Hypoglykämieneigung ${ }^{8)}$. Des Weiteren haben u.a. auch Früh- und Mangelgeborene ein erhöhtes Hypoglykämierisiko, weil sie nur geringe Glykogenspeicher und wenig Körperfett haben, was über eine reduzierte Lipolyse zu einem niedrigen Ketonkörperangebot für das Gehirn führt. Ausserdem zeigen diese Kinder eine unzureichende Glukoneogenese angesichts niedriger Blutzuckerspiegel, da ihnen eine ausreichende Menge an Substraten wie Laktat, Pyruvat, Alanin oder Ketonkörpern fehlt. Aus diesem Grunde sind routinemässige Blutzuckerbestimmungen bei Risikokindern notwendig (s. Abb. 1, Neugeborene mit einem erhöhten Risiko für neonatale Hypoglykämie) $)^{3,5,9-15)}$.

Die Frühernährung mit Muttermilch fördert die Glukoneogenese durch Bereitstellung notwendiger Substrate. Die Ketogenese wird durch Fettsäuren in der Milch angeregt, während es in Gegenwart von Laktose nur zu einem diskreten Anstieg des Insulinspiegels kommt. Im Gegensatz dazu kann die enterale Ernährung oraler Glukoselösungen zu erheblicher Insulin- und reduzierter Glukagonsekretion führen, was die Entwicklung eines Gleichgewichts aus Glukoneo- 


\section{Fortbildung}

genese und Ketogenese verzögert. Aus diesen theoretischen Gründen ist es vorteilhaft, eher Milch, als orale Glukoselösungen zu verabreichen ${ }^{3,9,10,14)}$.

Trotz jahrzehntelanger Forschung existiert bisher kein Konsens über die Definition der neonatalen $\mathrm{Hy}$ poglykämie. Es gibt mindestens vier Konzepte zur Definition von Blutzuckergrenzen, die auf den folgenden Kriterien beruhen: i) Klinische Symptome (sogenannte symptomatische Hypoglykämie); ii) Epidemiologische Daten; iii) Akute metabolische, endokrine und neurologische Befunde; iv) Neurologische Langzeitentwicklung.

Keine dieser Definitionen ist für sich alleine betrachtet ideal, und alle haben teils erhebliche Mängel 2,3,10,12,15-20). Die SGN sieht daher von einer spezifischen Definition der neonatalen Hypoglykämie ab und legt stattdessen Interventionsgrenzen für Prävention und Therapie der neonatalen Hypoglykämie fest.

Der genaue Effekt neonataler Hypoglykämie(n) auf die neurologische Langzeitentwicklung ist weiterhin unklar. Neueren Daten zufolge sind insbesondere spät nachweisbare Folgen möglich: In einer prospektiven Kohortenstudie an 528 Neugeborenen mit einem Gestationsalter $\geq 35$ SSW, die primär aufgrund von mütterlichem Diabetes mellitus rekrutiert wurden, fanden die Autoren bei der 2-Jahres-Kontrolle zunächst keinen Unterschied in der neurologischen Entwicklung von Kindern mit vs. ohne neonatale $\mathrm{Hy}$ poglykämie (Blutzucker $<2.6 \mathrm{mmol} / \mathrm{L}$ ) ${ }^{21)}$. Allerdings fielen in derselben Kohorte, bei Nachuntersuchung im Alter von 4.5 Jahren nach neonataler Hypoglykämie gehäuft eingeschränkte visomotorische und exekutive Funktionen auf, insbesondere bei Kindern, die schwere $(<2.0 \mathrm{mmol} / \mathrm{L})$, wiederholte, oder klinisch asymptomatische Hypoglykämien gehabt hatten ${ }^{22)}$. In einer weiteren, populationsbasierten Studie wurde die neurologische Langzeitentwicklung von 1'500 Neugeborenen ab 34 SSW nach moderater Hypoglykämie (<2.2 mmol/L) mit jener von 99'500 Neugeborenen ohne Hypoglykämie im Alter von 2 bis 6 Jahren verglichen. In dieser Kohorte war nach neonataler Hypoglykämie das adjustierte Risiko für die drei Parameter jegliche Entwicklungsverzögerung, motorische Entwicklungsverzögerung und kognitive Entwicklungsverzögerung jeweils erhöht ${ }^{23)}$. Eine kürzlich erschienene, randomisierte Studie fand bei Vergleich einer Interventionsgrenze von $2.0 \mathrm{mmol} / \mathrm{L}$ vs. 2.6 $\mathrm{mmol} / \mathrm{L}$ bei gesunden Neugeborenen ab $35 \mathrm{SSW}$ keine entwicklungsneurologischen Unterschiede im Alter von 18 Monaten $^{24}$. Allerdings liegen hierzu keinerlei Langzeitergebnisse vor. Angesichts o.g. Evidenz, dass entwicklungsneurologische Probleme als Folge einer neonatalen Hypoglykämie unter Umständen erst im Vorschulalter erkennbar sind, raten wir aktuell davon $\mathrm{ab}$, einen Schwellenwert von $2.0 \mathrm{mmol} / \mathrm{L}$ als Interventionsgrenze zu definieren.

\section{Differentialdiagnose}

Eine neonatale Hypoglykämie kann aus unterschiedlichsten Gründen auftreten, z.B. aufgrund einer gestörten Anpassung postnataler Stoffwechselprozesse, aber auch als unspezifisches Symptom einer Vielzahl von Erkrankungen (z.B. Infektion, Asphyxie, Polyzythämie, u.v.a.). Hypoglykämien sind sehr häufig bei Früh- und/oder Mangelgeborenen s. Leitlinie Betreuung von Frühgeborenen $34+0$ bis $36+6$ SSW www.neonet.ch/recommendations ${ }^{25}$. In den meisten Fällen lässt sich der Hauptgrund für die Hypoglykämie durch Anamnese und klinische Untersuchung herausfinden (z.B. Frühgeburtlichkeit, intrauterine Wachstumsverzögerung, maternaler Diabetes) ${ }^{26}$. Im Falle wiederholter oder persistierender Hypoglykämien trotz ausreichendem Angebot von Milch und Dextrose-Gel 40\%, sollten Neugeborene rasch zur i.v.-Glukosetherapie und weiteren Diagnostik auf eine Level II- oder Level III-Neonatologie verlegt werden, um Störungen wie einen persistierenden Hyperinsulinismus, andere Endokrinopathien, oder Stoffwechselerkrankungen auszuschliessen ${ }^{27}$.

\section{Diagnostische Überlegungen}

Die Anforderungen an Geräte zur neonatalen Blutzuckermessung sind hoch: Die Messergebnisse sollen bei minimaler Blutmenge unmittelbar erhältlich, kostengünstig und genau sein, letzteres insbesondere bei niedrigen Werten. Goldstandard ist die enzymatische Blutzuckerbestimmung im Labor mit der Hexokinasemethode. Allerdings kann eine verzögerte Bestimmung im Labor auch zu einem präanalytischen Absinken des Blutzuckerspiegels um ca. $0.3 \mathrm{mmol} / \mathrm{L} / \mathrm{h}$ durch Erythrozyten-Glykolyse führen ${ }^{28)}$. Aus praktischen Gründen werden daher häufig kleine, am Patientenbett einsetzbare, tragbare Geräte (bedside-Methode) verwendet. Die Genauigkeit dieser Geräte im Verhältnis zur Hexokinase-Methode wurde in vielen Studien untersucht ${ }^{29-35)}$. Zusammenfassend lässt sich sagen, dass die meisten Geräte den Blutzuckerspiegel überschätzen. Je nach Gerät liegt die Abweichung bei 0.2 bis $0.6 \mathrm{mmol} / \mathrm{L}$, wenn der Referenz-Blutzucker im Bereich von 2.0 bis $2.5 \mathrm{mmol} / \mathrm{L}$ ist. Andererseits kann es bei bedside-Methoden gelegentlich auch zu falsch-niedrigen Werten kommen. Da diese jedoch üblicher Standard zur Blutzuckermessung in Gebärsälen und auf Wochenbettstationen sind, sollte o.g. Messungenauigkeit bedacht werden. Unerlässlich ist die regelmässige Kalibration und Qualitätskontrolle der bedside-Methode. Auch sollte ein neues Gerät immer dann, wenn es in die Klinik eingeführt wird, mit der Hexokinase-Methode an Blutproben von Neugeborenen kalibriert werden ${ }^{36)}$.

\section{Blutzucker-Interventionsgrenze}

Idealerweise würde man die neonatale Hypoglykämie abhängig vom klinischen Kontext individuell definieren. Allerdings wären Sicherheit und Umsetzbarkeit eines solchen Vorgehens im klinischen Alltag äusserst fragwürdig. Aus pragmatischen Gründen hat sich in der klinischen Praxis daher die Nutzung einer fixen Interventionsgrenze - unabhängig von Bestimmungsmethode und Gerät - etabliert. Neurologische Langzeitprobleme scheinen aktuellen Daten zufolge hauptsächlich von der Blutzuckerstabilität in den ersten 48 Lebensstunden abzuhängen, d.h. vom Anteil der Blutzuckerwerte ausserhalb eines zentralen Bandes von 3 bis $4 \mathrm{mmol} / \mathrm{L}$, während die Hypoglykämie an sich 


\section{Fortbildung}

(Blutzucker < $2.6 \mathrm{mmol} / \mathrm{L}$ per Hexokinase-Methode) nicht mit entwicklungsneurologischen Einschränkungen im Alter von 2 Jahren verbunden war, sofern bei dieser Schwelle eine rasche Therapie erfolgte ${ }^{21}$. Da viele Kliniken primär mit der bedside-Methode arbeiten, die den Blutzuckerspiegel potentiell überschätzt, wird die Interventionsgrenze der neonatalen Hypoglykämie in dieser SGN-Leitlinie wie folgt festgelegt:

Ein Blutzuckerwert $<2.6 \mathrm{mmol} / \mathrm{L}$ bei Neugeborenen $\geq 35+0$ SSW spricht bei jeglicher Bestimmungsmethode für eine Hypoglykämie und muss behandelt werden.

Schema zur Prävention einer Hypoglykämie bei Neugeborenen mit erhöhtem Risiko

Die Abbildung 1 zeigt die empfohlene Prävention (Frühernährung, Dextrose-Gel 40\%, Zusatzernährung), sowie Blutzuckertests und weitere Massnahmen bei Neugeborenen mit einem erhöhten Risiko für Hypoglykämie. Ergänzend sollte der Energieverbrauch des Kindes minimiert werden, indem die Körpertemperatur im Normalbereich gehalten wird (s. Leitlinie Betreuung von Frühgeborenen $34+0$ bis $36+6$ SSW www.neonet.ch/recommendations ${ }^{25}$.

Schema zur Überwachung und Behandlung von Neugeborenen mit V.a. Hypoglykämie Die Abbildung 2 zeigt den Ablauf der Blutzuckertests und Massnahmen bei Neugeborenen mit hypoglykämieverdächtigen Symptomen.
Folgende Situationen müssen vermieden, bzw. bei Auftreten schnell und entschlossen behoben werden, weil sie mit einem erhöhten Risiko für neurologische Einschränkungen verbunden sind, ${ }^{22}$ auch sollte rasch Kontakt mit einer Neonatologie aufgenommen werden, um das weitere Vorgehen abzustimmen:

- Schwere Hypoglykämie $<2.0$ mmol/L

- Prolongierte Hypoglykämie > 4 Stunden

- Wiederholte Hypoglykämie

\section{Erklärungen zu den Fussnoten} in den Schemata

\section{Erhöhtes Risiko für Hypoglykämie}

- Frühgeborene < 37+0 SSW

- Mütterlicher Diabetes (sowohl diätetisch eingestellt, als auch insulinpflichtig)

- Geburtsgewicht < 2500 g am Termin oder bei Geburtsgewicht $<3$. Perzentile

- Geburtsgewicht > $4500 \mathrm{~g}$ am Termin oder bei Geburtsgewicht $>97$. Perzentile

- Kranke Neugeborene (z.B. Asphyxie, Infektion, Atemnotsyndrom, Hämolyse, u.v.a.)

- Hypothermie $\left(<36.5^{\circ} \mathrm{C}\right)$

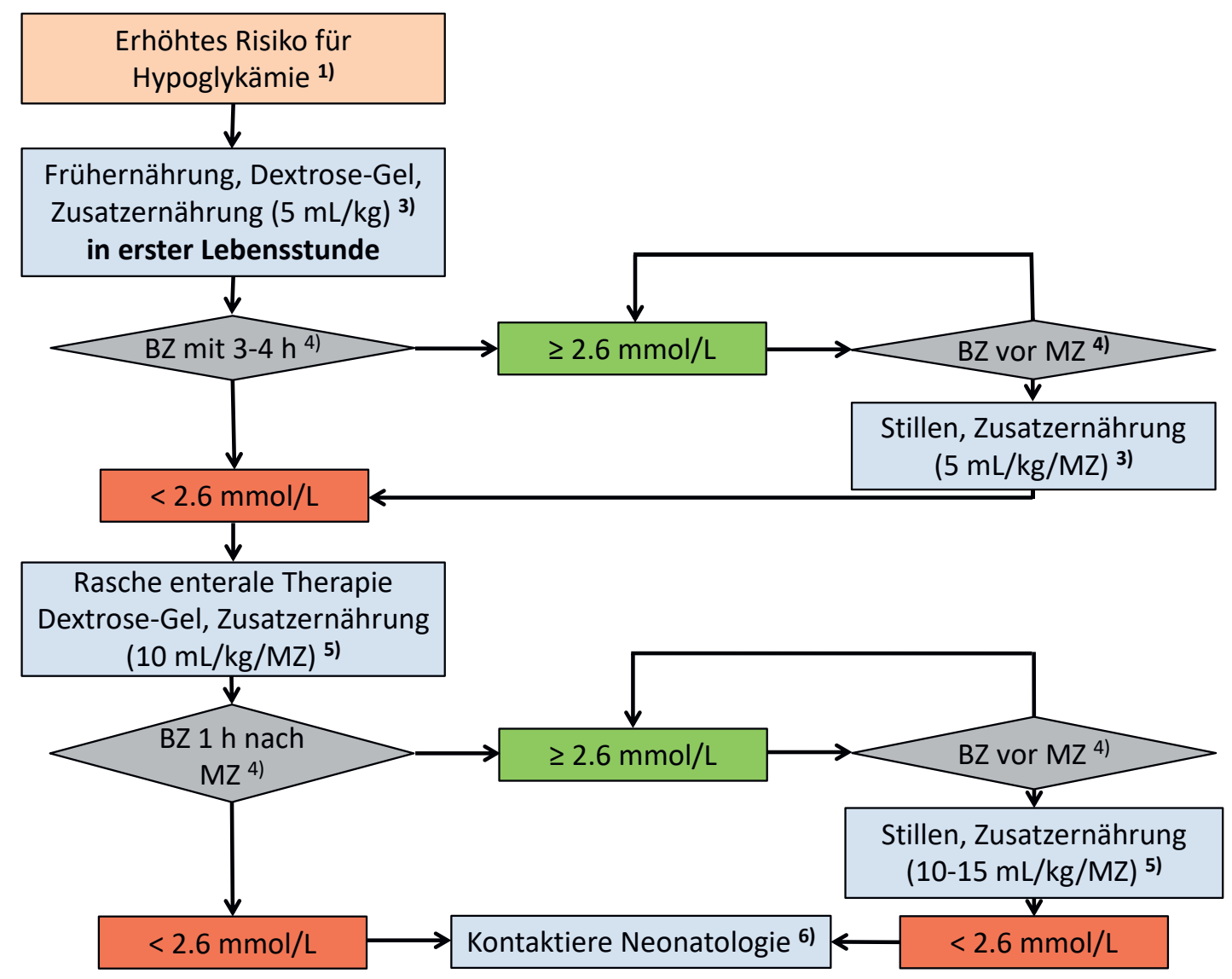

Abbildung 1: Erklärungen der Fussnoten s. Seiten 7 und 8; (BZ = Blutzucker, MZ = Mahlzeit). 


\section{Fortbildung}

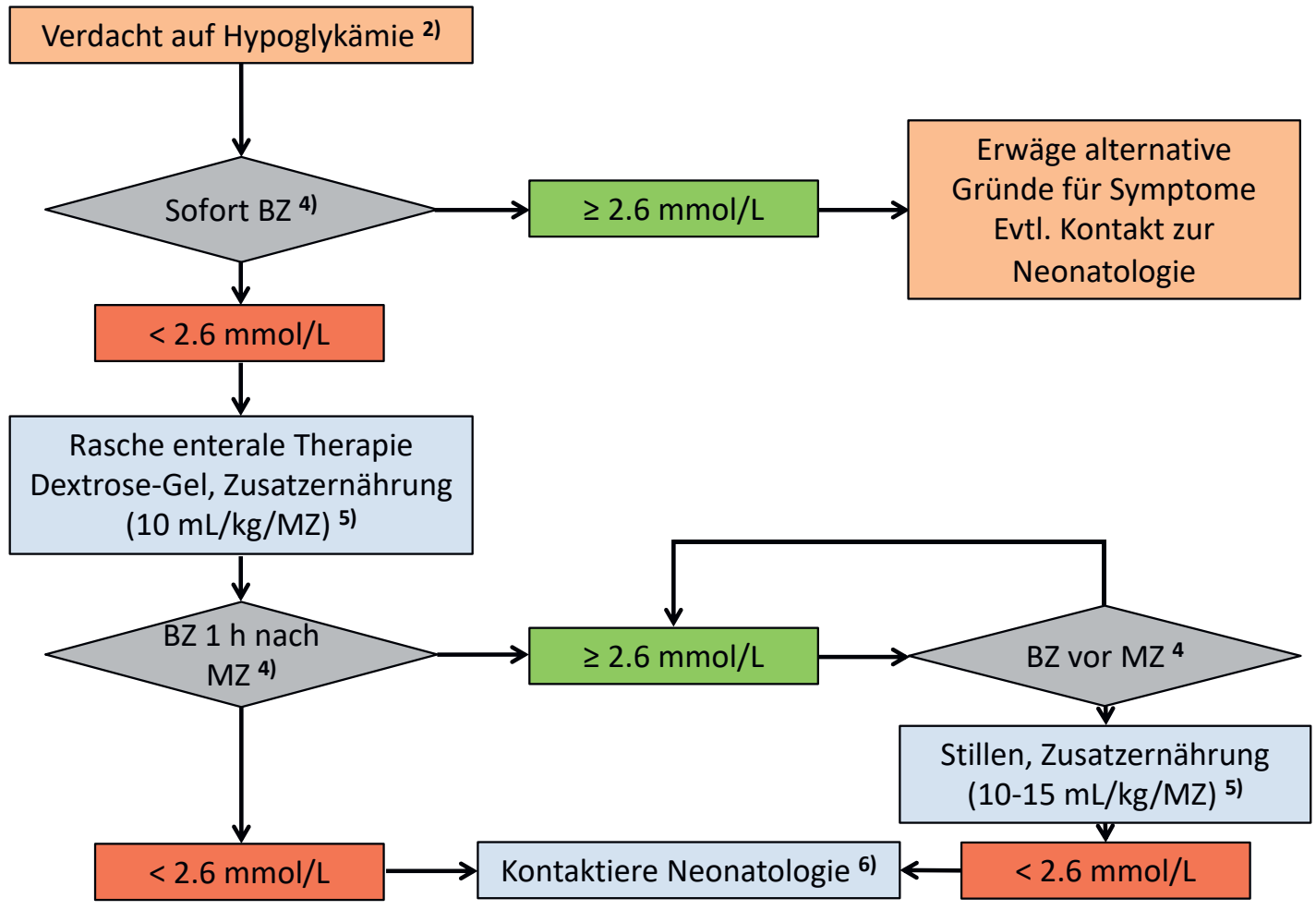

Abbildung 2: Erklärungen der Fussnoten s. Seiten 7 und 8; (BZ = Blutzucker, MZ = Mahlzeit).

\section{Verdacht auf Hypoglykämie}

Hypoglykämieverdächtige Symptome können neurologisch (Muskelhypotonie, Hyperexzitabilität, Apathie, Krampfanfälle), kardiorespiratorisch (Apnoe, Zyanose, Blässe, Tachykardie, Bradykardie), oder sonstiger Art sein (z.B. Schwitzen, Tremor, Zittrigkeit, Hypothermie). Bei neurologischen Symptomen aufgrund einer Hypoglykämie geht man davon aus, dass das Gehirn zu wenig Glukose und alternative Energieträger erhält, daher ist eine schnelle und effektive Behandlung nötig. Hypoglykämiesymptome sind unspezifisch, deswegen sollten bei Persistenz der Symptome trotz normalisiertem Blutzucker alternative Diagnosen in Erwägung gezogen werden.

\section{Frühernährung, Dextrose-Gel $40 \%$, \\ Zusatzernährung}

\section{Pränatale Kolostrumgewinnung}

In manchen Kliniken wird Schwangeren die vorgeburtliche Kolostrumgewinnung angeraten. Dies erfolgt unter der Annahme, nach der Geburt Kolostrum bei Kindern mit erhöhtem Risiko für Hypoglykämie als Zusatzernährung zu verabreichen, um den Blutzuckerspiegel anzuheben, bzw. es zur Behandlung manifester Hypoglykämie einzusetzen. Leider spricht die Datenlage dafür, dass pränatal gewonnenes Kolostrum fast immer nur in geringer Menge exprimiert wird und nach Geburt praktisch keinen Effekt auf den Blutzuckerspiegel hat ${ }^{37)}$. Andererseits gibt es keine Evidenz dafür, dass dieses Vorgehen schadet und es könnte die Kuhmilchexposition in den ersten Lebenstagen reduzieren ${ }^{38)}$.

\section{Frühernährung mit Muttermilch}

Neugeborene mit erhöhtem Risiko für Hypoglykämie sollten unmittelbaren Hautkontakt mit ihrer Mutter haben und möglichst früh an die Mutterbrust ange- legt werden (spätestens innerhalb der ersten Lebensstunde). Während der ersten 2 bis 3 Lebenstage sollten sie alle 2 bis $3 \mathrm{~h}$ gestillt werden.

\section{Prophylaktische Gabe von Dextrose-Gel 40\%}

Eine Einzeldosis von 200 mg/kg KG Dextrose-Gel 40\% ( $0.5 \mathrm{~mL} / \mathrm{kg} \mathrm{KG}$ ), im Alter von 1 Lebensstunde in die Wangenschleimhaut massiert, senkt das Risiko für eine neonatale Hypoglykämie. Dies gilt für Kinder mit erhöhtem Risiko für Hypoglykämie und ist nicht auf Neugeborene diabetischer Mütter beschränkt ${ }^{39}$.

\section{Formulamilch}

Unmittelbar nach dem Stillen kann dem Kind zusätzlich Formulamilch angeboten werden ( $5 \mathrm{~mL} / \mathrm{kg} \mathrm{KG}$ ), bis ausreichend Muttermilch zur Verfügung steht.

\section{Blutzuckerbestimmungen}

Der Blutzucker kann mit bedside-Methoden gemessen werden. Bei asymptomatischen Neugeborenen mit erhöhtem Risiko für Hypoglykämie sollte der erste Blutzucker im Alter von 3 bis 4 Lebensstunden bestimmt werden, d.h. unmittelbar vor der 2. Mahlzeit. Im Falle einer Hypoglykämie sollte er nach $1 \mathrm{~h}$ wiederholt werden, um den Behandlungserfolg zu überprüfen. Falls der Kontrollwert $\geq 2.6 \mathrm{mmol} / \mathrm{L}$ ist, sind trotzdem weitere Messungen vor den nächsten Mahlzeiten notwendig. Sobald drei aufeinanderfolgende Messungen normal sind, kann auf weitere Bestimmungen verzichtet werden. Bei hypoglykämieverdächtigen Symptomen muss der Blutzucker unmittelbar gemessen werden.

\section{Enterale Therapie}

Die Diagnose einer Hypoglykämie erfordert eine rasche Behandlung ohne jegliche Verzögerung. Eine Ein- 
zeldosis 200 mg/kg KG (0.5 ml/kg KG) Dextrose-Gel $40 \%$ sollte zügig und unmittelbar vor dem Stillen in die Wangenschleimhaut einmassiert werden, weil es die Chance für einen Behandlungserfolg verbessert, die Verlegung auf die Neonatologie verhindern kann, die Stillrate im Vgl. zu Placebo erhöht ist, die neurologische Entwicklung nicht beeinträchtigt wird und es zudem kostengünstig ist ${ }^{40,41}$. Dextrose-Gel $40 \%$ kann in den ersten 48 Lebensstunden wiederholt gegeben werden, wobei im Falle wiederholter Hypoglykämien immer ein Neonatologe oder Pädiater zugezogen werden sollte. Innerhalb der ersten 2 bis 3 Lebenstage sollte im Anschluss an 2- bis 3-stündliches Stillen zusätzlich Formulamilch angeboten werden (ca. $10 \mathrm{~mL}$ / $\mathrm{kg} \mathrm{KG}$ ), so dass man auf eine Gesamtmenge von etwa 10-15 mL/kg KG pro Mahlzeit kommt. Im Allgemeinen ist von oralen Glukoselösungen (z.B. Glukose 10\%) abzuraten. Bei Ernährungsschwierigkeiten kann, entsprechende Kompetenz vorausgesetzt, die Ernährung über eine Magensonde erwogen werden, wobei hier ein Neonatologe oder Pädiater beratend zugezogen werden sollte.

\section{Kontaktaufnahme mit der Neonatologie}

Wenn eine Hypoglykämie durch enterale Ernährung und Dextrose-Gel $40 \%$ nicht behoben werden kann, ist eine i.v.-Therapie notwendig. Derartige Behandlungen finden üblicherweise auf Neonatologien der Stufe Ila, Ilb, oder III gemäss CANU-Einteilung statt ${ }^{1}$. Im Falle schwerer, prolongierter, oder wiederholter Hypoglykämien ist eine rasche Kontaktaufnahme mit der nächstgelegenen Neonatologie dringend angeraten, um die weiteren Massnahmen zu besprechen.

Für das Literaturverzeichnis verweisen wir auf unsere Online Version des Artikels.

\section{Autoren}

Prof. Dr. med. Sven Schulzke, Abteilung Neonatologie, Universitäts-Kinderspital beider Basel UKBB

Dr. med. Seema Das-Kundu, Zürich

Jehudith Fontijn, Klinik für Neonatologie, Universitätsspital Zürich

Dr. med. Marion Mönkhoff, Kindermedizin, Klinik Hirslanden Zürich

Dr. med. Roland Neumann, Abteilung Neonatologie, Universitäts-Kinderspital beider Basel UKBB

PD Dr. med. Gabor Szinnai, Abteilung Diabetologie und Endokrinologie, Universitäts-Kinderspital beider Basel UKBB

Die Autoren haben keine finanziellen oder persönlichen Verbindungen im Zusammenhang mit diesem Beitrag deklariert. 